\title{
IdeAs
}

Idées d'Amériques

\section{Comment le tourisme permet-il la valorisation du secteur du cacao ? L'exemple de La République dominicaine et du Costa Rica}

¿Cómo el turismo permite la valorización del sector del cacao? El ejemplo de la República Dominicana y de Costa Rica

How does tourism allow the valorization of cacao? The example of the Dominican Republic and Costa Rica

\section{Laura Henry}

\section{OpenEdition}

\section{Journals}

Édition électronique

URL : https://journals.openedition.org/ideas/4174

DOI : $10.4000 /$ ideas.4174

ISSN : 1950-5701

Éditeur

Institut des Amériques

Référence électronique

Laura Henry, «Comment le tourisme permet-il la valorisation du secteur du cacao ? L'exemple de La République dominicaine et du Costa Rica », IdeAs [En ligne], 12 | 2018, mis en ligne le 19 décembre 2018, consulté le 19 octobre 2022. URL : http://journals.openedition.org/ideas/4174 ; DOI : https:// doi.org/10.4000/ideas.4174

Ce document a été généré automatiquement le 19 octobre 2022

\section{(c) (i) (9)}

Creative Commons - Attribution - Pas d'Utilisation Commerciale - Pas de Modification 4.0 International - CC BY-NC-ND 4.0

https://creativecommons.org/licenses/by-nc-nd/4.0/ 


\title{
Comment le tourisme permet-il la valorisation du secteur du cacao? L'exemple de La République dominicaine et du Costa Rica
}

\author{
¿Cómo el turismo permite la valorización del sector del cacao? El ejemplo de la \\ República Dominicana y de Costa Rica \\ How does tourism allow the valorization of cacao? The example of the Dominican \\ Republic and Costa Rica
}

Laura Henry

\section{Introduction}

1 Le Costa Rica et la République dominicaine ont tous deux développé un secteur touristique considérable: le Costa Rica est la principale destination touristique d'Amérique centrale (Boukhris L., 2013:5) et la République dominicaine celle de la Caraïbe (Dehoorne O. et al., $2010: 3$ ). Mais plus qu'un secteur de l'économie nationale, le tourisme est un outil d'affichage de ces sociétés, le Costa Rica se construisant comme une «nation verte» (Boukhris L., 2013: 5) alors que la République dominicaine s'est longtemps représentée comme une destination balnéaire « sol y playa ».

2 En effet, le tourisme de plage est l'activité principale du secteur touristique de la République dominicaine, qui a développé le modèle des hôtels tout-inclus (Alvarado J. and Oddone N., 2017 : 247). L'imaginaire lié à la Caraïbe repose effectivement sur les stéréotypes des plages de sable blanc, aux eaux cristallines et bordées de cocotiers, et la République dominicaine n'y déroge pas (Dehoorne 0., 2007: 3). Les complexes balnéaires, desservis par les charters, sont la traduction même du tourisme de masse ; ils sont centralisés autour de Punta Cana et de Puerto Plata (Auzias D. et Labourdette J. P. 2011 : 9). 


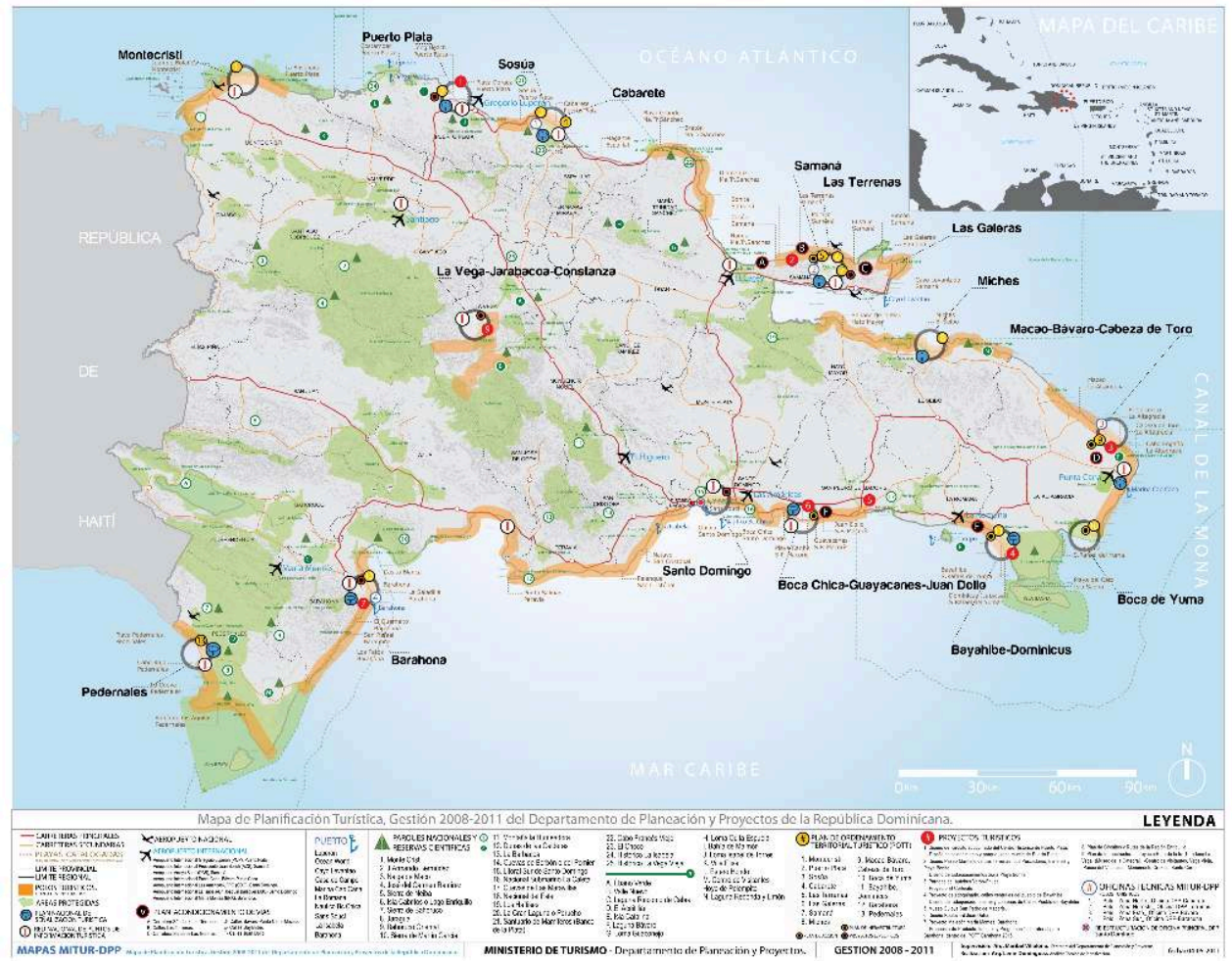

Concernant le Costa Rica, la nation s'est clairement positionnée comme étant le modèle $\mathrm{du}$ tourisme vert avec une offre touristique diversifiée qui repose sur les parcs nationaux et les activités de nature et d'aventure. Ce processus de construction d'un patrimoine national naturel s'effectue dès le XIX ${ }^{e}$ siècle et se consolide dans la décennie 1990, notamment par les politiques de promotion mise en place de l'Institut costaricien du tourisme (ICT) qui visent à la fois une clientèle nationale et internationale (Boukhris L., 2016: 266). Paradoxalement, bien que l'imaginaire associé au Costa Rica soit la nature sauvage, il existe tout de même de grandes infrastructures et un tourisme de plage important, notamment à Jaco et Tamarindo (Morera C., $2006: 3$ ).

Figure 2 : Carte touristique du Costa Rica

Blanco M., 2007 


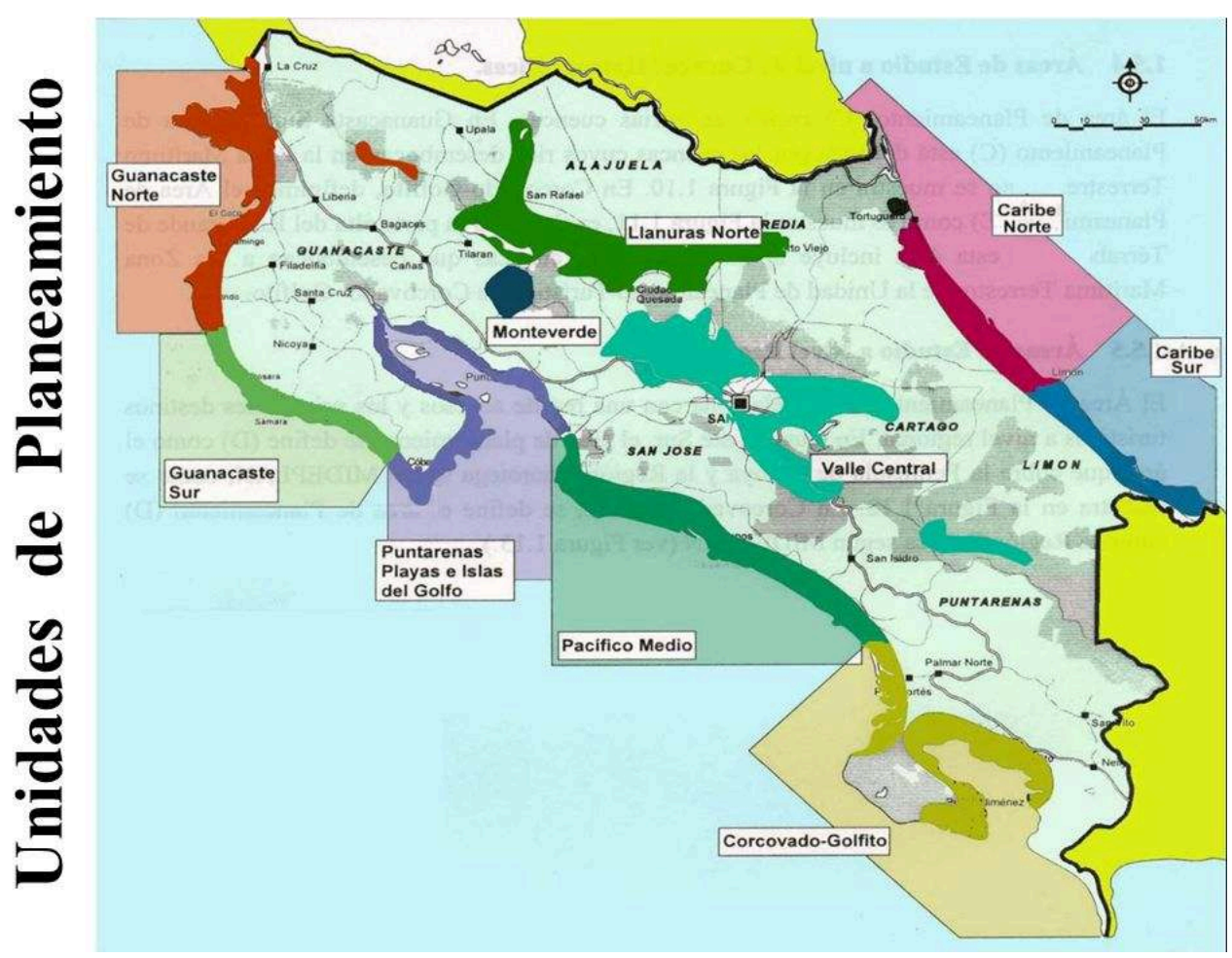

Dans les deux cas, on observe depuis une dizaine d'années une transition vers un tourisme affiché comme plus durable. Récemment, les deux pays ont intégré une dimension culturelle et identitaire avec le développement d'un tourisme rural et culturel. La protection de l'environnement est néanmoins au cœur des politiques de développement comme en témoigne le développement de nombreux parcs nationaux et d'aires protégées. Ces deux pays misent sur une diversification de leur offre vers le tourisme durable, notamment avec la patrimonialisation de certaines productions agricoles emblématiques, ou « rendues » emblématiques. À l'instar du café en Colombie (Lotero Contreras J., et al 2008 : 179), des cigares à Cuba (Herrera R., 2012: 59) ou encore du vin en France (Debos F., 2008 ; Jacquet O. et Laferté G., 2013), le cacao est mis en tourisme en République dominicaine et au Costa Rica sous la forme de parcours pédagogiques et gustatifs. Cet article propose d'analyser les cas spécifiques de la mise en tourisme du cacao en République dominicaine et au Costa Rica et les dynamiques engendrées par ce tourisme grâce à l'étude de données empiriques récentes. À travers le cacao, il est intéressant d'analyser les mutations dont le tourisme est à la fois moteur et sujet au sein de ces deux pays. Quelles sont les stratégies de mises en tourisme du cacao en République dominicaine et au Costa Rica et dans quelle mesure sont-elles liées au développement de la filière cacao dans ces deux pays? Bien que la production de cacao costaricienne ne représente qu'un seul pourcent de la production dominicaine (700 tonnes en 2014 pour le Costa Rica contre 70000 tonnes sur l'île caraïbéenne (FAO, 2017)), l'intérêt de la comparaison repose d'une part sur la dimension touristique et patrimoniale du cacao mise en place au Costa Rica de manière quasi automatique, en opposition à une production plus "conventionnelle » en République dominicaine, et d'autre part sur les trajectoires touristiques affirmées et différenciées de ces deux pays du bassin caraïbéen.

5 La méthodologie utilisée pour cette réflexion repose sur une démarche itérative recourant aux observations de terrain, aux entretiens avec les acteurs locaux, à la 
cartographie ainsi qu'aux outils complémentaires de l'agriculture comparée et du diagnostic agraire ${ }^{1}$. Ces données empiriques ont été récoltées en 2011 et 2015 en République dominicaine et en 2017 et $2018^{2}$ au Costa Rica. C'est à partir du prisme d'une recherche sur la patrimonialisation du cacao qu'est analysé le tourisme autour du cacao en République dominicaine et au Costa Rica.

\section{Un positionnement commun de la République dominicaine et du Costa Rica : la valorisation de la filière du cacao par la qualité}

6 Le marché du cacao est ancré dans un contexte éminemment concurrentiel dominé par le continent africain qui produit à lui seul $66,5 \%$ de la production mondiale (FAO, 2014). L'Amérique latine produit quant à elle $15,7 \%$ du cacao (ibid.). La stratégie commerciale prédominante du continent est de se différencier sur les marchés internationaux grâce à un cacao de bonne qualité, ancré sur un territoire particulier. Il s'agit alors de créer une qualification territoriale à l'échelle d'un pays à laquelle contribue la mise en tourisme du cacao. Mais dans le cas de nos deux pays d'étude, c'est également à travers le tourisme que la qualité du cacao est mise en lumière. La qualité du cacao est différenciée sur les marchés internationaux en cacaos courants et cacaos fins, en fonction de leurs qualités physiques et organoleptiques (Loor Solórzano, R. G., $2007: 1$ ) ainsi que de leur origine géographique (par pays) (Despréaux D. et Leblond A., 1996: 115). Le potentiel aromatique des fèves dépend des conditions du milieu, des variétés utilisées, des modes de cultures et des traitements post-récolte. C'est principalement la fermentation et le séchage qui influent sur la qualité organoleptique des fèves (Barel M., $2013: 1$; Guehi T. et al., $2010: 2509$; Kanmogne A. et al., $2012: 94$; Dulcire M. et Roche G., 2006: 13) ${ }^{3}$. Chaque pays cherche à se différencier grâce à la construction d'une image de marque qui peut être mise en valeur par le tourisme.

\section{Le changement de paradigme de la République dominicaine}

7 Jusqu'à la fin des années 1980 , le cacao dominicain avait une mauvaise réputation sur le marché international, malgré un bon potentiel car sa production n'était pas standardisée et la fermentation inexistante. Le cacao traditionnel de type Sanchez, non fermenté, possède un beurre plus dur, et donc de qualité moyenne, est commercialisé et exporté majoritairement vers les États-Unis. Dans les années 1980, afin d'améliorer la qualité du cacao ainsi que sa renommée internationale, le Président Guzman appuie la création d'organisations de petits producteurs (Kessari Dea Edgaar M.-E., 2008: 25), soucieuses d'augmenter leurs bénéfices en améliorant la qualité du cacao (Couve $\mathrm{C}$. et Croguennec L., 2000). Sous l'impulsion du gouvernement, associations de producteurs, chercheurs, organismes d'exportations et institutions ont œuvré pour réaliser un véritable changement de paradigme de la filière du cacao en promouvant la qualité grâce à la mise en place d'un processus de fermentation homogène donnant le cacao de type Hispaniola, aujourd'hui considéré comme cacao fin (Roche G., 2006: 13; ICCO, 2015). Le cacao Hispaniola est rattaché à son origine, à savoir le territoire dominicain. On peut donc parler d'un ancrage territorial du produit, qui se retrouve dans la construction touristique autour du cacao. 
Photographies 1 et 2 : Caisses de fermentation et récupération du cacao fermenté après 5 jours, Conacado, Hato Mayor

Laura Henry et Catherine Taillon Desrochers, 2011
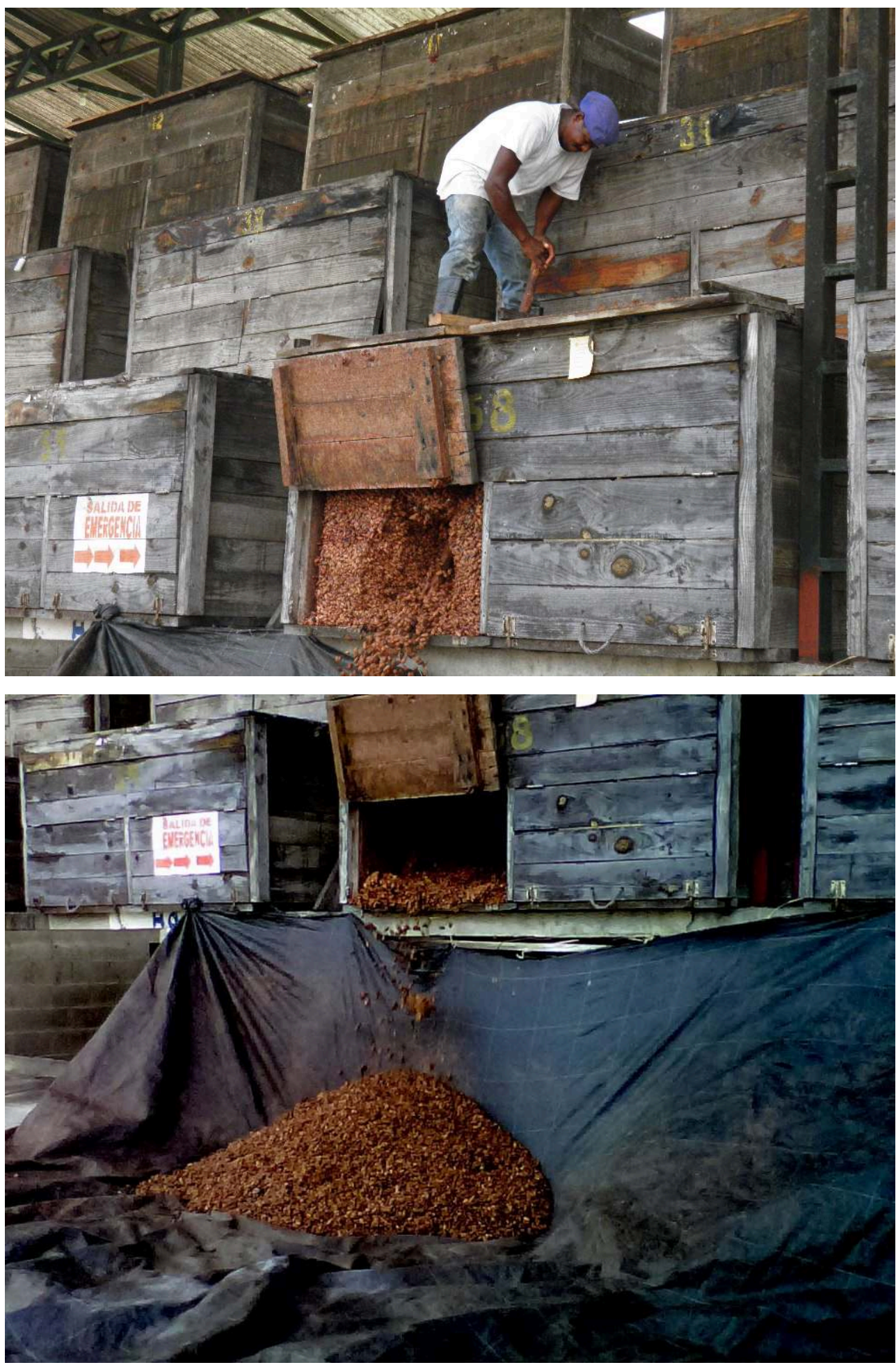

8 De plus, la République dominicaine a su se positionner comme le leader en cacao biologique sur le marché international. Elle est le premier exportateur et producteur mondial de cacao biologique, avec 15000 tonnes exportées en 2010 (Ramirez P. E., 
2013: 8). Cette stratégie de différenciation sur les marchés de niche contribue à augmenter la visibilité de la République dominicaine à l'international.

9 La République dominicaine est l'un des cinq plus gros pays producteurs de cacao certifiés commerce équitable (CE) par l'organisme certificateur Fairtrade Labelling Organisation (FLO, 2011). Les stratégies de développement agricole de la filière mettent en avant la protection de l'environnement grâce à l'agroforesterie, le caractère territorial et patrimonial du cacao dominicain mais également l'allégorie de la petite paysannerie afin d'asseoir le caractère éthique de cette culture. Cela se retrouve a fortiori dans la mise en tourisme du cacao.

La carte page suivante représente les dynamiques territoriales de la filière cacao dominicaine. Elle illustre les zones de production et situe les infrastructures des cinq principales organisations exportatrices.

Figure 3 : Répartition territoriale de la filière cacao en République dominicaine

Crédit : Laura Henry

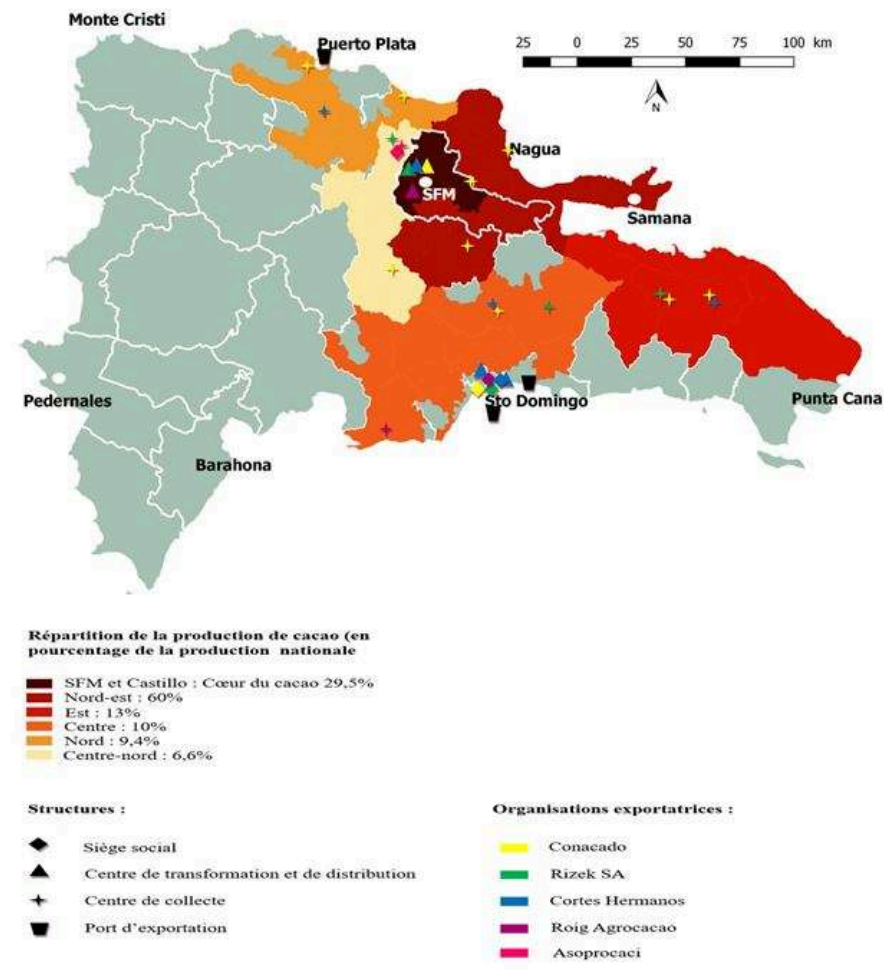

\section{La relance de la culture par le Costa Rica}

La situation est bien différente au Costa Rica. La production costaricienne est considérablement moins importante: elle représente environ $1 \%$ de celle de la République dominicaine. Cela s'explique en partie par le fait que le Costa Rica a été touché par la Moniliasis, maladie causée aux cacaoyers par le champignon Moniliophthora roreri (Krauss U. et al., 2003: 53). Entre 1978 et 1982, $80 \%$ de la production a été perdue, ce qui en fait la maladie la plus dévastatrice pour le cacao. Elle est responsable des charnières historiques du cacao costaricain. En effet, l'avant Moniliasis a correspondu à une période concentrée sur l'augmentation de la 
production, tandis que l'après Moniliasis a favorisé le développement de techniques de résistance aux maladies et l'amélioration de la qualité afin de renforcer la compétitivité sur les marchés d'un produit rare mais reconnu. C'est le Centre de Recherche et d'Enseignement d'Agronomie Tropicale (CATIE) qui, grâce à son programme d'amélioration génétique du cacao, a permis de créer de nouvelles variétés plus résistantes à la maladie et de bonne qualité ${ }^{4}$.

Les cours du cacao ayant remonté ces dernières années, la production a été relancée. En 2011, le Costa Rica a signé la Convention internationale du cacao afin de renforcer le secteur cacaoyer national et d'appuyer son développement (Tribunal Supremo de Elecciones, 2016). Ainsi, la filière est partie intégrante des politiques agricoles mises en place par le ministère de l'Agriculture et de l'Élevage costaricien ${ }^{5}$. La politique agricole concernant le cacao met en avant la pratique de l'écotourisme, le caractère patrimonial de cette culture ainsi que des systèmes de culture en agroforesterie, respectueux de l'environnement. La qualité du cacao costaricien est indéniable; toute la production relève de la catégorie "cacao fin" (ICCO, 2015). Dans le contexte d'un volume de production peu important, la stratégie de commercialisation et de valorisation du cacao se base sur la qualité, jouant sur différents facteurs et principalement les facteurs environnementaux, sociaux et organoleptiques.

Dans les politiques publiques, la relance de la culture du cacao est présentée comme une alternative aux monocultures de banane et d'ananas très présentes au Costa Rica, permettant ainsi de réduire les risques d'inondations et la vulnérabilité économique liés à la monoculture (Dirección Regional Huerta Atlantica, 2011:3). Le secteur cacaoyer tente de s'implanter sur des marchés de niches : cacao fin et cacao biologique. La stratégie de développement du secteur est basée sur le positionnement du pays comme cacao de qualité, stratégie partagée par les acteurs publics et privés (La Nación, 2016).

Figure 4 : Carte de la répartition de la production de cacao au Costa Rica 


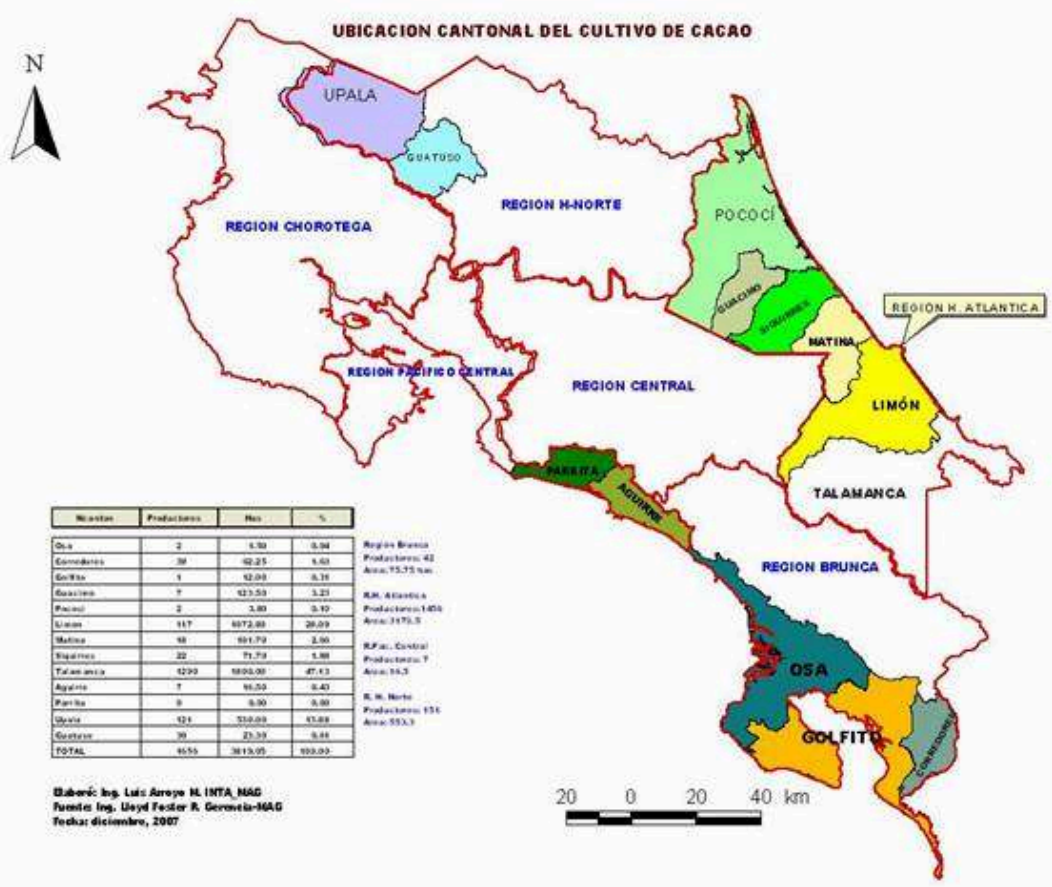

14 L'intérêt du Costa Rica comme objet d'étude est la dimension touristique et patrimoniale du cacao, qui est mise en place institutionnellement, dans une optique de réactivation de la production (Ibid.). La République dominicaine emploie une stratégie de valorisation à plus grande échelle, mais également en jouant sur l'amélioration de sa qualité, notamment à travers la production biologique et certifiée CE. Dans les deux cas, la production est assurée principalement par de petites exploitations familiales, avec une dimension identitaire cependant plus prononcée au Costa Rica car une grande partie des producteurs sont indigènes et afro-descendants. Il est intéressant à présent d'analyser les stratégies de mise en tourisme du cacao des deux pays et les dynamiques qu'elles engendrent.

\section{Le tourisme comme vitrine de la qualité du cacao}

Le tourisme est un objet d'étude géographique à part entière, étudié souvent pour en mesurer les impacts et faire émerger de nouveaux modèles, dans une logique de durabilité bien que le champ des études sur le tourisme soit bien plus large. Pour Requier-Desjardins (2010: 97), la qualification du produit touristique renvoie au processus de qualification territoriale et selon Lazzarotti (2011), de façon générale, les activités créées à partir de la valorisation du territoire sont dans une grande partie des cas directement ou indirectement liées au tourisme, les nouveaux lieux et offres touristiques sont créés pour augmenter l'attractivité des territoires et par là favoriser le développement économique (Idir M. S, 2013 : 5). Il ne s'agit pas dans cet article de réaliser un état de l'art sur la question mais de resituer le contexte d'étude dans un champ conceptuel. La littérature francophone et hispanophone distingue différentes formes de tourisme : écologique, durable, rural, de masse, de croisière... bien que ces catégories soient à prendre avec recul car elles peuvent ne pas être considérées comme des catégories scientifiques. Néanmoins, nous retiendrons les travaux de Dehoorne et de ses confrères $(2007,2010,2014)$ ainsi que ceux de Breton (2009) et de Sarrasin (2016). 
La littérature anglophone s'apparente aux tourism studies et elle est assez productrice, notamment avec des revues spécialisées ${ }^{6}$. Nous retiendrons également la thèse de Boukhris (2013), qui met en parallèle l'imaginaire touristique et l'imaginaire national, et souligne l'importance des choix politiques et idéologiques qui ont guidé la trajectoire très spécifique du Costa Rica.

16 Le type de tourisme qui nous intéressera tout particulièrement est ce que RequierDesjardins qualifie de tourisme "alternatif" qui prend en compte le tourisme rural, l'écotourisme et encore le tourisme communautaire (2010). Plus précisément, la mise en tourisme du cacao peut être qualifiée de tourisme agro-alimentaire; ce dernier consiste en une offre touristique autour des produits et des productions agroalimentaires de terroir (Blanco M., 2007, 2010, 2012 ; Acuña M. et Ruiz K., 2000 ; Barrera E. et Bringas Alvarado O., 2009 ; Castellanos E., 2014). La littérature latinoaméricaine est abondante en études de cas, et ce travail de recherche propose d'enrichir et d'actualiser les données empiriques liées à ce type de tourisme. Le tourisme rural vient également s'inclure dans un système agroalimentaire localisé permettant la diversification de l'offre et la valorisation du produit. Pour la littérature francophone, nous retenons De Myttenaere (2011) ainsi que Moizi-Maïzi et Bouche (2011) qui s'intéressent aux patrimoines agroalimentaires européens.

\section{Le Costa Rica : la volonté politique d'utiliser le tourisme du cacao comme levier de développement de la filière cacao}

Suite aux ravages causés par la Moniliasis, la production de cacao costaricien a été quasi unanimement délaissée. La relance du cacao s'est opérée progressivement à partir des années 2000. Le gouvernement ne s'est impliqué dans la filière qu'à partir de 2002, avec la volonté de réactiver la filière cacao grâce au Programme National mais le programme ne voit réellement le jour qu'en 2006 (Llach Cordero L., 2007 : 3). En signant l'Accord international du $\mathrm{cacao}^{7}$ en 2011, le gouvernement costaricien a affirmé son intérêt pour la filière (Tribunal Supremo de Elecciones, 2016). Le secteur privé s'organise avec la création de la Chambre du Cacao, qui voit le jour en 2010.

L'intérêt du tourisme dans le développement des politiques publiques se retrouve tout particulièrement dans le plan de développement du cacao de la région Huertar Caribe. Ce dernier met en évidence l'importance du potentiel touristique de la culture et l'intérêt de la pluriactivité pour les producteurs, notamment grâce au tourisme (Dirección Regional Huerta Atlántica, 2011: 16). L'argumentaire développé permet d'affirmer que le ministère de l'Agriculture et de l'Élevage et le ministère du Tourisme envisagent la filière cacao à travers le prisme de la qualité et du tourisme. Le gouvernement encourage les initiatives de production à être accompagnées d'une mise en tourisme, et associées également si possible à une identité ethnique spécifique, indigène ou afro-descendante.

Dans cette perspective, le cacao a été reconnu comme patrimoine national du Costa Rica en 2015 (décret $\mathrm{N}^{\circ} 39230-\mathrm{C}-\mathrm{MAG}$ ). La production, la transformation, la commercialisation et l'exportation du cacao et de ses dérivés ont été déclarées d'intérêt national. Une journée nationale du cacao a été décrétée le 4 juillet de chaque année (Gobierno de Costa Rica, 2015 : 5). Il est intéressant de noter ici comment le cacao n'est plus seulement perçu à travers le prisme commercial ni celui de la qualité mais comment la dimension culturelle est prise en compte dans la déclaration du cacao en 
tant que patrimoine national. Le cacao est reconnu pour son importance sociale, économique et religieuse depuis l'époque précolombienne (Gasco J., 1997: 155), toujours présente pour les populations indigènes qui le considèrent comme un aliment sacré et divin (Hébert A. et Ammae-Khodja P., 1999 : 4 ; Entretiens réalisés en mai 2018 dans la communauté indigène de Bribri). Concernant l'héritage afro-descendant, bien que la fourchette temporelle soit plus courte (environ 150 ans), les revendications culturelles sont palpables puisque le cacao a été pendant de nombreuses années l'une des cultures principales des descendants d'immigrants jamaïcains.

\section{La République dominicaine : visibilité à l'international et stratégie de promotion}

Dans le cas de la République dominicaine, il est pertinent de s'attarder sur la mise en visibilité d'une telle activité. Deux des plus grands organismes exportateurs du pays, la coopérative CONACADO et l'entreprise Rizek SA ont créé des parcours du cacao, visant à montrer tout le processus de production suivant le principe beans to bar, respectivement appelés le Tour del chocolate (Clearco Gí, 2011a, 2011b) et El sendero del cacao (Rizek SA, 2016). En plus d'accueillir des touristes locaux et internationaux, ces organismes utilisent leurs prestations touristiques afin de démontrer de la qualité de leurs produits à un client potentiel. Ces excursions sont ainsi souvent proposées aux membres d'institutions internationales ou du gouvernement dominicain lors de la négociation de certains financements, générant ainsi une sorte de tourisme institutionnel. Il s'agit de démontrer la qualité du cacao in vivo grâce aux facteurs de qualité écologiques, sociaux et organoleptiques propres au cacao dominicain.

Photographie 3 : Tour del Chocolate, Présentation de la culture du cacao dans une plantation, Hato Mayor del Rey, République dominicaine

Laura Henry, 2011

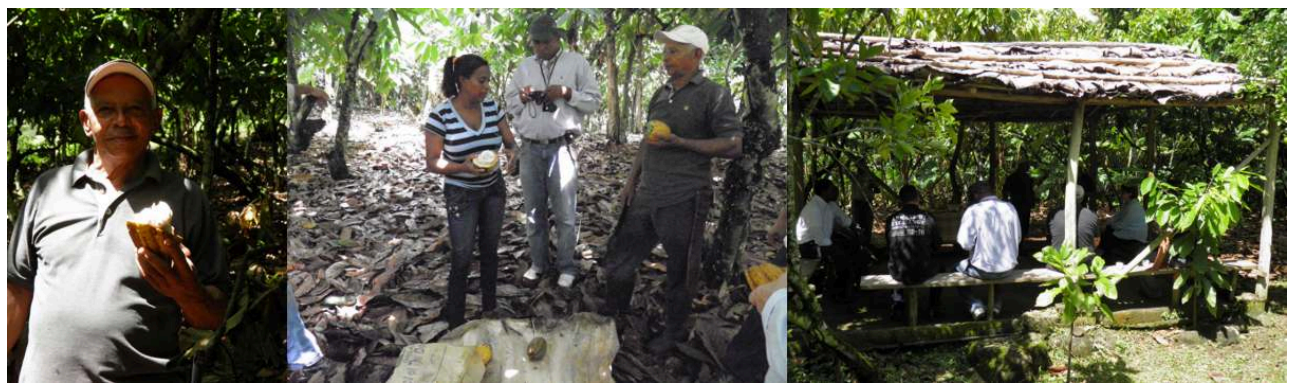

Photographie 4 : Tour del Chocolate, Présentation de la fermentation et du séchage à la succursale de la CONACADO, Hato Moyor del Rey, République dominicaine 


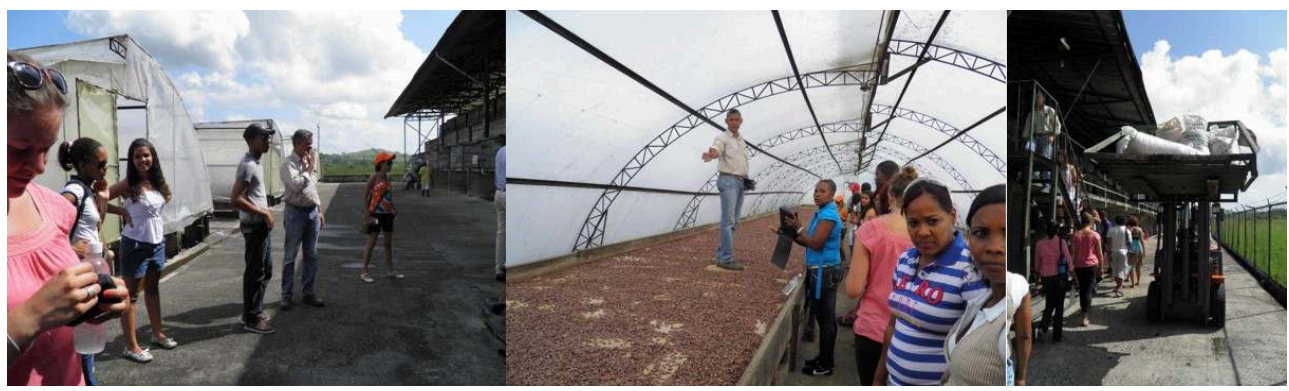

Photographie 5 : Présentation de la transformation artisanale du cacao en chocolat par Elana Vega lors de la Ruta del Café, parcours écotouristique autour de la production de café, de cacao et de caoutchouc, Samána, République dominicaine

Laura Henry, 2015

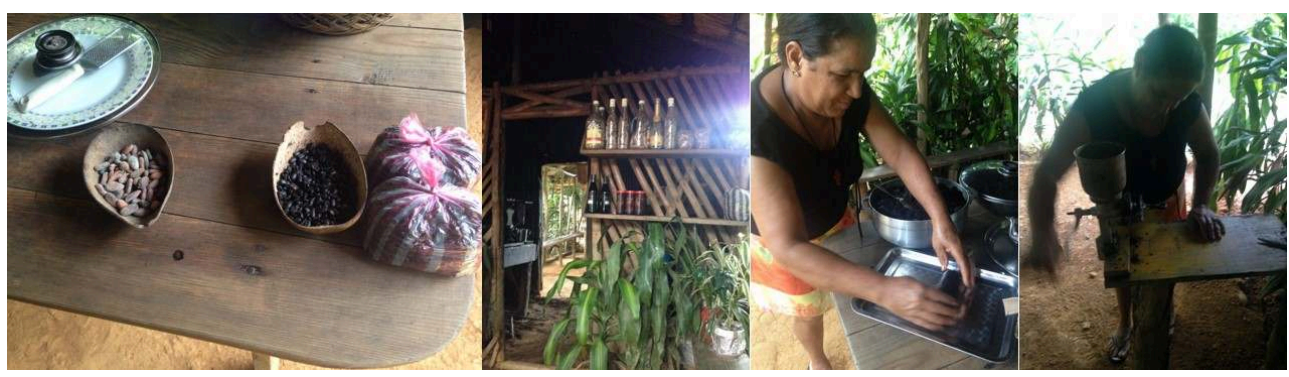

De plus, grâce aux médias, aux réseaux sociaux et à internet, les touristes ayant réalisé les tours diffusent des articles ou des photos et se font ainsi les promoteurs de la qualité du cacao dominicain mise en évidence lors des excursions (Griotte, 2013). Ce processus de diffusion moderne de l'information contribue à associer la République dominicaine à un nouveau type de tourisme et à renforcer son image et sa présence sur la toile.

Le tourisme rural contribue ainsi à l'éloge d'une qualification territoriale du cacao expliquant que la qualité du produit repose sur la maîtrise des " écosystèmes cultivés » (Requier-Desjardins D., 2013: 3): un système dans lequel les savoir-faire et les caractéristiques écologiques du milieu et donc l'origine territoriale attribuent des caractéristiques spécifiques au produit. Touzard et Vandecandelaere (2005) cités par Requier-Desjardins (2013:3), notent que la valorisation des produits « typiques » est de plus en plus liée à l'activité touristique. Dans ce cadre-là, les parcours écotouristiques permettent une proximité géographique avec les potentiels acheteurs, leur permettant de percevoir les qualités non intrinsèques du produit. Un touriste peut, en plus des achats qu'il va effectuer sur place, être ensuite plus attentif à l'origine du cacao qu'il va consommer au quotidien. Il pourra alors développer une sorte de lien affectif avec le cacao dominicain dont il a pu appréhender la production et la transformation. Concernant les acheteurs directs des organisations exportatrices, ces parcours permettent de créer une telle proximité géographique. L'écotourisme autour du cacao sert également à faire l'apologie de la qualité du cacao dominicain. Il est instrumentalisé par la Conacado et Rizek SA pour mettre en valeur le cacao dominicain auprès des acheteurs et des organisations internationales. Cela profite par rayonnement au gouvernement dominicain, dans la mesure où les secteurs du cacao et du tourisme ont une place importante dans le développement du pays ${ }^{8}$. Lors de la Conférence mondiale sur le cacao organisée en mai 2016, certains des participants étrangers ont pu réaliser les parcours de la Conacado et de la Rizek (Entretiens réalisés 
au sein de la Conacado et de la Rizek en juin-juillet 2015). Ces participants étaient principalement des représentants d'acheteurs ou des membres d'institutions.

Au Costa Rica comme en République dominicaine, les facteurs contribuant à la « qualité sociale ${ }^{9}$ " du cacao sont particulièrement valorisés par les parcours écotouristiques proposés, généralement grâce aux historiques des organisations, qu'elles soient de type associatif ou privé. L'image du petit producteur est toujours mise en avant, en mettant l'accent sur les valeurs culturelles et sociales de la culture du cacao. En fonction des types d'acteurs en charge du tour, ce sont différents aspects qui sont mis en valeur. Par exemple, les coopératives ou les entreprises montreront souvent les avantages générés par l'affiliation à leur structure, notamment le prix versé aux producteurs, les prestations sociales qu'ils assurent ou encore les moyens et les infrastructures mis à disposition, afin de démontrer le compromis éthique auquel ils s'engagent. Au Costa Rica, dès que la dimension ethnique (indigène ou afro-descendante) est présente, elle est mise en lumière en expliquant l'importance du cacao pour les cultures concernées. En République dominicaine, l'agroforesterie et ses bénéfices environnementaux, économiques et sociaux sont presque toujours mis en valeur. Chaque offre touristique participe ainsi à la construction du patrimoine autour de la culture du cacao, en promouvant le caractère culturel, environnemental et social. Néanmoins, se soulève alors la question des risques qu'une telle instrumentalisation de la culture locale peut engendrer, des conséquences de sa folklorisation sur les populations locales qui doivent alors rester dans un imaginaire correspondant aux attentes des touristes, obligées de prétendre ou de jouer un rôle pour y répondre.

Photographie 6 : Edgar Campbell, Président de la coopérative Afrocacao lors d'un circuit éducatif dans sa plantation, Puerto Viejo, Costa Rica

Laura Henry, 2018

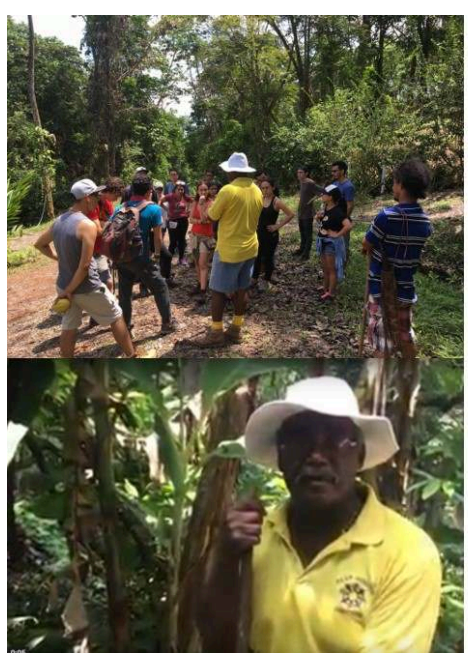

"Queremos reconstruir los valores que nos hicieron un pueblo económicamente independiente. Queremos fomentar la solidaridad, ser amos del cacao y no esclavos del cacao. Mejorar el valor agregado para mejorar la calidad de vida de los productores. Que la producción de cacao y el turismo permita un tejido social para rearmar y reorientar las relaciones ya que el cacao permite unir la cuenca alta-media-baja y la frontera".

Edgar Campbell, entretien du 18 mai 2018

Photographie 7 : Circuit proposé par l'association ACOMUITA (Asociación Comisión de Mujeres Indígenas de Talamanca), mettant en avant les connaissances ancestrales indigènes à Bribri, au Costa Rica

Alexa Obando, 2018 


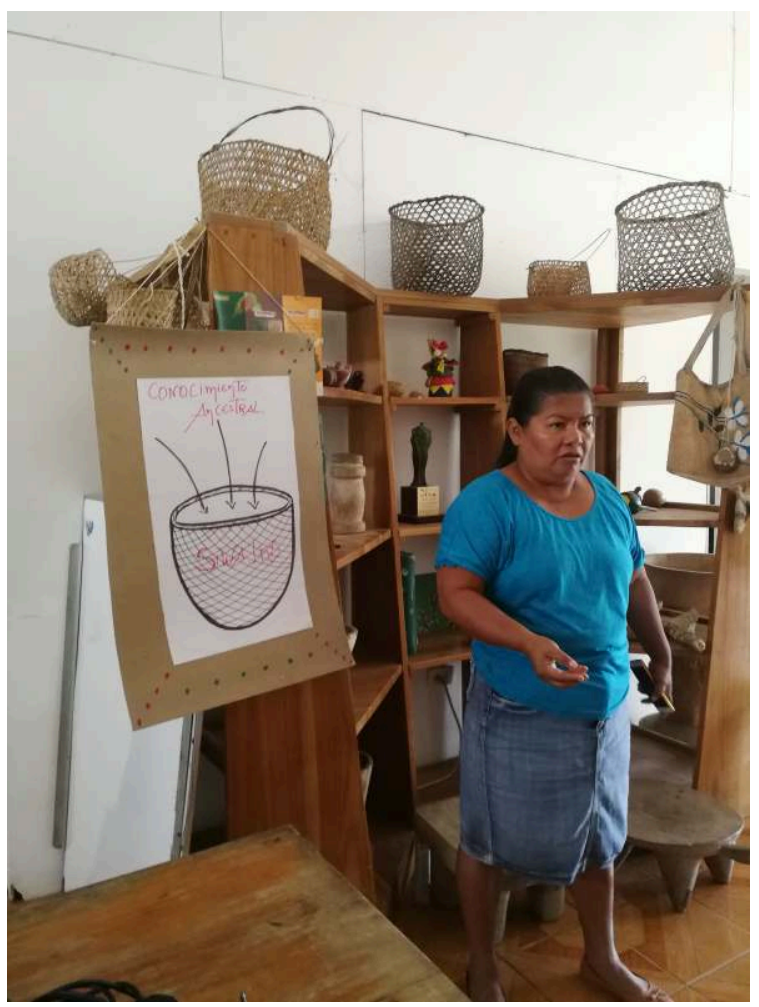

Il est important de noter que la pluriactivité engendrée dans ces deux pays par les parcours touristiques autour du cacao est génératrice de revenus non négligeables pour les producteurs et les intermédiaires ${ }^{10}$. Les tours proposés durent en moyenne une matinée et se terminent généralement par un repas local. Ils sont souvent accompagnés de la vente directe de produits transformés et d'artisanat grâce aux boutiques de proximité. Au Costa Rica, le prix d'un circuit de ce type est compris entre $25 \$$ et 30 \$. Ces tours reposent sur des initiatives privées ou associatives de petite envergure. Il existe une prolifération d'offres dans différentes régions du Costa Rica. Dans le canton de Talamanca, plus d'une dizaine de parcours différents sont proposés. En République dominicaine, le prix du tour varie entre $30 \$$ et $35 \$$. Deux sont organisés par des organismes d'exportations (déjà cités) et il existe également quelques initiatives privées de producteurs. Dans les deux cas, bien que des prestataires intermédiaires assurent bien souvent l'organisation et la réalisation du circuit, une part non négligeable revient malgré tout aux acteurs les plus défavorisés de la filière: les producteurs et les productrices. Il convient à présent de s'intéresser aux transformations engendrées par la mise en tourisme du cacao au sein des deux pays.

\section{L'effet boomerang du tourisme, débats émergents dans les sociétés dominicaines et costariciennes}

\section{La place des femmes révélée}

La mise en tourisme du cacao a pour conséquence une plus grande visibilité des femmes impliquées dans la filière. En effet, bien que la production de cacao soit un secteur qui continue à être considéré comme une "affaire d'homme " (World Cocoa Foundation, n.d.), la part des femmes dans ce secteur est considérable. Il est possible de les 
catégoriser en trois groupes : les femmes de producteurs; les femmes productrices, à savoir des femmes référentes et propriétaires de la plantation; et les femmes ouvrières agricoles (UTZ certified 2009: 6). En République dominicaine, la majorité des femmes qui travaillent dans les plantations le font de manière non-rémunérée, comme membre de la famille et comme travail occasionnel (Barrientos S., 2013: 7). Leur travail n'est alors pas reconnu à leur juste valeur.

Photographie 8 : Tour del Chocolate, Présentation de la transformation du cacao en chocolat, en vin de cacao et en confiture par l'association de femmes Mujeres Esperanzas Unidas, qui peuvent bénéficier de l'espace pour vendre leur produit et préparer le repas, Hato Mayor

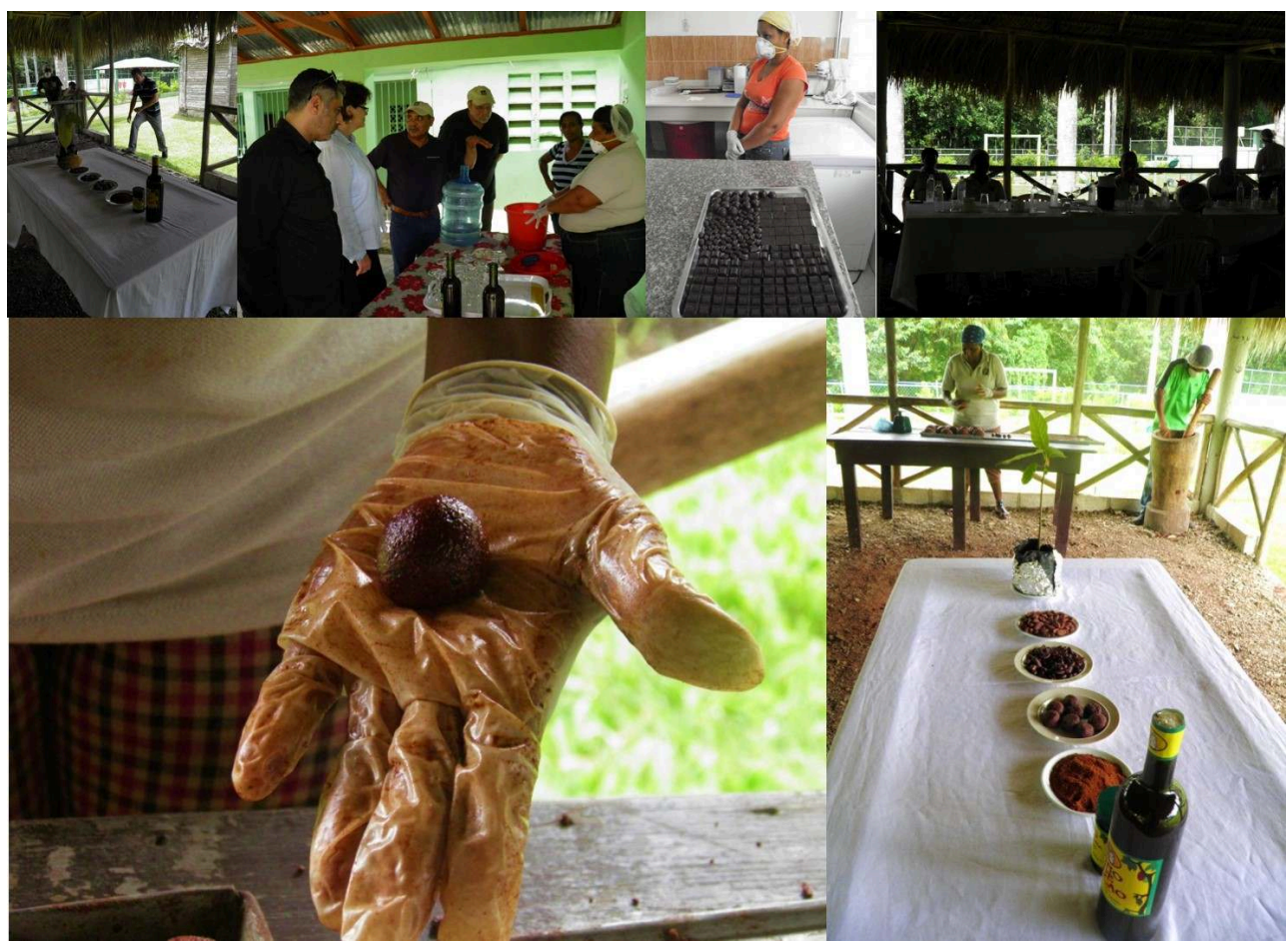

Au Costa Rica, la tradition matrilinéaire du peuple indigène Bribri, présent sur la côte caraïbe, fait que les femmes sont les propriétaires foncières des plantations, et ce sont elles qui s'occupent également des parcours écotouristiques. C'est le cas de l'association ACOMUITA (Asociación Comisión de Mujeres Indígenas de Talamanca) et de l'association APPTA (Asociación de Pequeños Productores de Talamanca) dont 38 \% des associés sont des femmes (entretien avec la présidente de l'association, mai 2018). Malheureusement, seule la région de Huertar Caribe compte autant de femmes dans ses producteurs, dans les autres cas, le Costa Rica rejoint la République dominicaine dans l'invisibilisation des femmes de la filière et les observations confirment les observations de la littérature existante sur la place de la femme dans le secteur cacaoyer en Côte d'Ivoire, au Ghana et en Inde (Fair Labour Association, 2015 ; Barrientos S., 2013 : 7 ; Oxfam, 2013 ; World Cocoa Foundation, 2016; UTZ certified 2009). Les épouses participent aux travaux agricoles, notamment en période de récolte. Les tâches qu'elles réalisent sont majoritairement l'écabossage et la séparation des grains, l'entretien des cultures vivrières, la préparation de plants, le nettoyage des plantations et le séchage du cacao (Barrientos S., 2013 :18). Une partie du salaire des ouvriers correspond aux petitsdéjeuners et aux déjeuners préparés et servis par les épouses et/ou les filles des 
exploitants, parfois pour une trentaine de personnes. En outre, dans le cas des producteurs et intermédiaires, la gestion des comptes est relayée par les deux membres $\mathrm{du}$ couple ou par leur fille.

En plus de leurs activités directement liées à l'exploitation familiale, les femmes ont en charge toute l'intendance du foyer et ont souvent une activité professionnelle extérieure qui contribue au revenu du ménage.

Photographie 9 : Portraits de femmes lors d'une réunion d'association rattachée à la CONACADO, Hato Mayor, République dominicaine
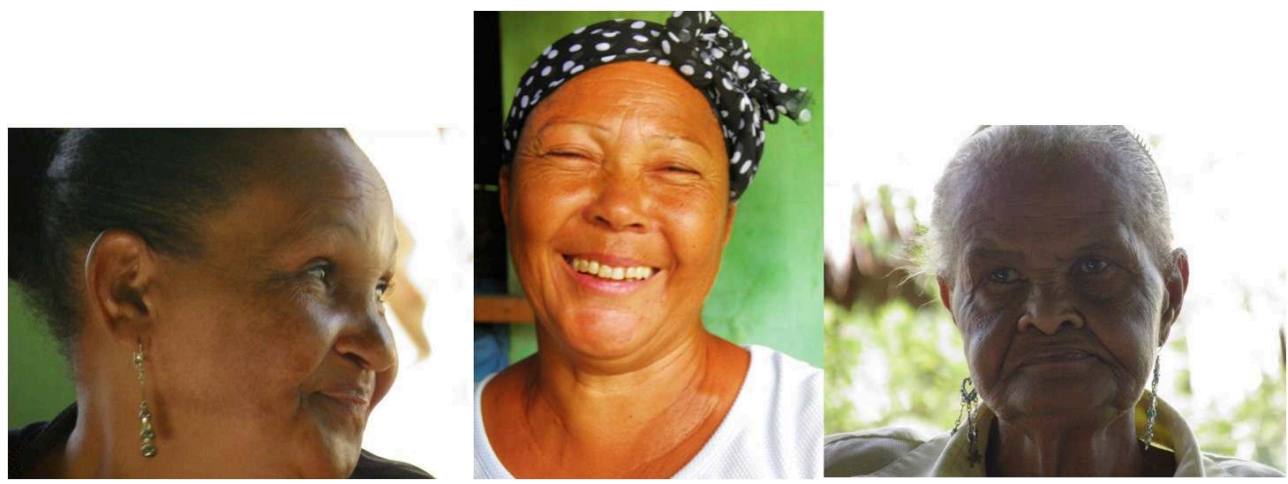

L'intérêt des parcours touristiques est la mise en lumière des associations de femmes, leur permettant de monétariser des taches qui ne le sont d'ordinaire pas, comme la préparation des repas.

L'intérêt des parcours touristiques est la mise en lumière des associations de

femmes, leur permettant de monétariser des taches qui ne le sont d'ordinaire pas, comme la préparation des repas.

Ils permettent également de libérer la parole en leur donnant un espace pour présenter leurs activités ainsi que des points de vente pour les nombreux produits élaborés qu'elles réalisent (chocolat, liqueur de cacao, confiture de cacao, artisanat, art). Dans le cas des indigènes Bribri, c'est un moment de partage et de transmission de leurs traditions ancestrales nécessaire à la pérennité de leur culture mais également un moyen de résistance à la culture machiste dominante : "Nous luttons contre le machisme extrême qui va à l'encontre de la culture Bribri et montrer notre culture et nos valeurs aide à diffuser l'idée d'un autre modèle de société» (Faustina Torres, 30 avril 2018). Dans le contexte actuel mondial et particulièrement latino-américain où l'un des enjeux sociétaux est l'amélioration de la condition féminine, chaque levier potentiel de développement à destination des femmes est à mettre en lumière et à valoriser mais également à critiquer afin de l'améliorer. De futures recherches permettront dans un second temps de venir étoffer cet article.

Photographie 10 et 11 : Feria de las Semillas, deux productrices de cacao, Bribri, Costa Rica

Laura Henry, mai 2018

IdeAs, 12 | 2018 


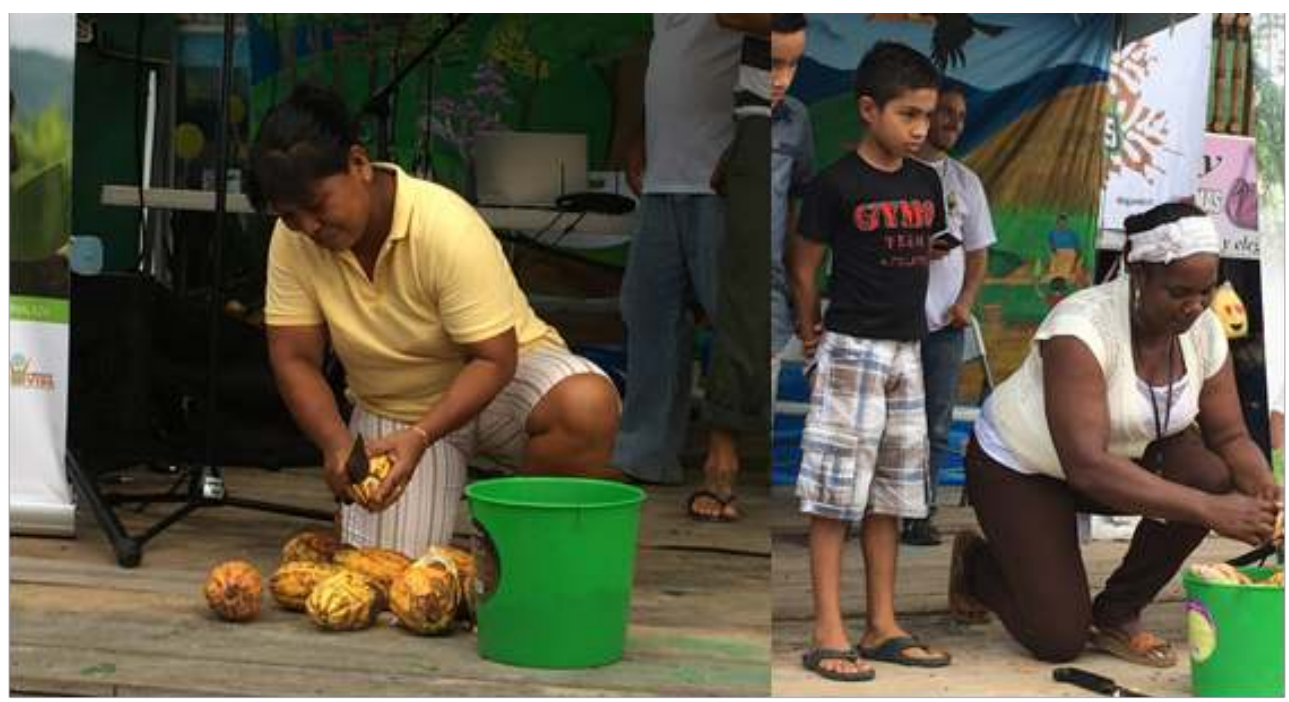

Photographie 12 : Vente d'art lors du Tour del Chocolate par l'association de femmes Mujeres Esperanzas Unidas, Hato Mayor del Rey, République dominicaine

Catherine Taillon Desrochers, 2011

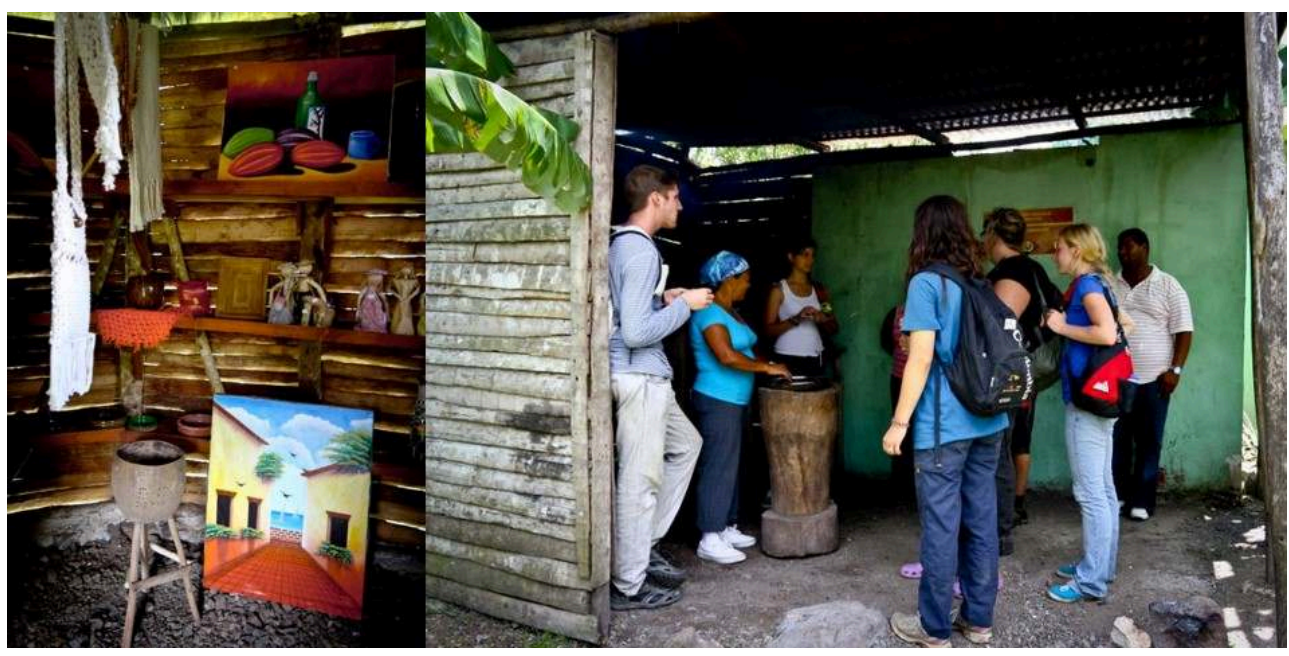

\section{Effets du tourisme du cacao sur les imaginaires touristiques nationaux}

Bien que le développement touristique du Costa Rica soit basé sur l'écotourisme (Boukhris, 2016), les activités proposées étaient principalement contrôlées par des personnes extérieures aux communautés locales ou par des opérateurs de tourisme de masse. Elles relevaient d'activité de "nature " incluant peu les populations. Les retombées économiques ne restaient pas aux au sein des communautés (AndreuBoussut V., 2008: 170). C'est à partir de 2002 que l'Institut du tourisme costaricien essaie de mettre l'accent sur le tourisme rural communautaire comme alternative de développement, et met à l'ordre du jour l'intérêt des populations rurales (ibid.). Des parcours agro-touristiques se sont multipliés depuis lors au Costa Rica (Blanco M., 2007 : 27), et notamment des tours du cacao. Un éventail hétérogène d'acteurs les propose; des organismes associatifs, des entreprises privées, des petits et grands 
producteurs, des chocolatiers, des locaux, indigènes, afro-descendants ou métis et encore des étrangers, souvent européens. Cette hétérogénéité se retrouve dans la façon dont les circuits sont organisés ou gérés. En effet, on retrouve des inégalités dans les moyens disponibles pour l'aménagement d'infrastructures, l'accès à un public international, souvent à cause de la barrière de la langue ou bien encore la difficulté à promouvoir le circuit, et dans l'« expérience touristique » pour répondre aux attentes des touristes. Par exemple, au Costa Rica, l'offre touristique de l'association ACOMUITA, bien que très intéressante grâce à la culture Bribri mise en avant, n'est pas disponible en anglais, les explications fournies ne sont pas organisées et construites pour un touriste non averti (observations réalisées lors d'un tour organisé pour un groupe d'étudiants en mai 2018) et le circuit est situé dans une zone un peu reculée, où seules des personnes véhiculées peuvent avoir accès (ACOMUITA, n.d.). Habituée aux projets de recherche et de développement, ACOMUITA reçoit principalement des groupes d'étudiants, de chercheurs ou bien d'ONG tandis que le tour proposé par l'entreprise Caribeans Chocolate, fondée par un étranger, située dans le village balnéaire de Puerto Viejo, en quasi bord de mer, est accessible et visible pour toute personne passant par le village. Toutes les informations sont disponibles en anglais, la boutique et le restaurant/bar sont dans l'esprit bohème chic et proposent même des cours de Yoga et de Pilates (Caribeans Chocolate, n.d.). De même, Chocolate Adventure Company a été fondée par un Costaricien ayant eu une carrière entrepreneuriale à la capitale avant de se tourner vers le cacao et sa valorisation touristique, sa vision, ses contacts et ses moyens sont clairement plus fournis que celle de personnes isolées ${ }^{11}$. Son infrastructure est située au port de la ville de Limon, en centre-ville, juste en face de la sortie des bateaux de croisière. Il propose un tour complètement adapté au format croisière, une explication et une expérience express, dans ses bâtiments où un circuit avec photos, vidéos, fabrication et dégustation est organisé. Les approches sont donc différentes en fonction des acteurs, en fonctions des moyens disponibles et des logiques enclenchées. Néanmoins, dans la majorité des cas, le touriste est placé au cœur de la vie locale le temps d'une visite. Il est intéressant de noter comment le tourisme rural permet une expérience touristique plus proche des gens. Dans certains cas, il permet également de faire un lien avec les dimensions culturelles et ethniques du pays, parce qu'il peut être mis en place par les populations les plus marginalisées (petite paysannerie, populations minoritaires) qui sont propriétaires des moyens de production et par conséquent détentrices de l'offre touristique (Morera C., 2006: 5). Elles peuvent alors revendiquer et partager leur identité et leur culture. C'est notamment à travers le tourisme rural que ces populations sortent de l'invisibilité à laquelle elles étaient confrontées dans la construction de l'imaginaire national, construit autour de la «blanchitude » et de la biodiversité rendant la pluralité ethnique et culturelle invisible (Boukhris L., 2013 : 9).

31 La République dominicaine propose, grâce à ce genre d'initiative, une offre touristique différente de ses attributs touristiques traditionnels, le tourisme de plage et de masse. En effet, le ministère du Tourisme dominicain cherche à diversifier son offre et à proposer un tourisme "durable» (Ministero de Turismo, 2018). Les initiatives de tourisme autour du cacao en République dominicaine viennent a priori s'opposer au tourisme de masse ${ }^{12}$ et montrer une autre facette de la République dominicaine en promouvant des régions peu visitées par le tourisme, comme la région de Hato Mayor et celle de San Francisco de Marcoris qui proposent les deux principaux tours du pays (Rizek S.A., 2016 ; Clearco Gí, 2011a). Néanmoins, afin de viser une clientèle plus large, 
ces initiatives cherchent à atteindre la clientèle des hôtels tout-inclus des stations balnéaires en démarchant les complexes hôteliers, et proposant des tours avec hébergement et transport. Ce faisant, elles viennent donc bousculer l'imaginaire touristique liée à la République dominicaine en lui donnant une dimension rurale, populaire et authentique. Mais au-delà de l'imaginaire touristique, c'est également l'imaginaire de la nation qui est transformé grâce au cacao. En effet, le changement de paradigme de la qualité du cacao, qui place la République dominicaine en tant que premier exportateur de cacao biologique au niveau mondial, ainsi que la promotion de la qualité grâce à l'utilisation des parcours touristiques comme vitrine ont permis de changer l'image de la République dominicaine sur la scène internationale, ajoutant à l'imaginaire qui lui est lié des dimensions environnementales, de ruralité et d'éthique.

\section{Conclusion}

La mise en tourisme du cacao se développant en République dominicaine et au Costa Rica est un cas d'étude spécifique de nouvelles pratiques de tourisme rural. Cet article a permis de mettre en lumière comment les filières cacao des deux pays utilisent le concept de qualité pour se positionner sur les marchés internationaux. Le Costa Rica s'appuie sur un cacao fin et une dimension ethnique afro-descendante et indigène, où le cacao est associé au territoire. La République dominicaine quant à elle a fait des efforts pour modifier son mode de production et améliorer l'image de son cacao. La mise en tourisme du cacao ou plus particulièrement de son mode de production est encouragée par le gouvernement dans le cas du Costa Rica. Ce dernier démontre de son intérêt pour le secteur cacao en décrétant le cacao comme patrimoine national. En République dominicaine, ce sont les organismes d'exportation qui impulsent cette mise en tourisme et entendent ainsi redorer l'image du cacao dominicain. Le tourisme est ici instrumentalisé comme une vitrine de la qualité du cacao.

Le tourisme permet également de révéler et de valoriser la place des femmes au sein des filières cacao des deux pays en leur conférant un rôle central dans la gestion de l'offre touristique et en permettant de rémunérer des travaux qui ont tendance à être invisibilisés. Il donne également une fenêtre d'action aux associations de femmes qui peuvent alors bénéficier de cet espace pour vendre leur produit mais également faciliter le partage culturel.

34 L'impact de ce type de tourisme est bien entendu critiquable, on peut se poser la question des effets négatifs d'une telle folklorisation et instrumentalisation de la culture sur les communautés locales. Néanmoins, au vu de la marginalisation des acteurs concernés, le bilan semble être positif permettant à la fois une pluriactivité des exploitations agricoles ainsi qu'un accès direct à des ressources financières non négligeables.

De plus, les mutations engendrées par la mise en tourisme du cacao contribuent à bousculer les imaginaires liés aux deux États en proposant de nouvelles offres touristiques permettant de révéler certains de leurs aspects méconnus. Le Costa Rica vient ici mettre en lumière son multiculturalisme et son « humanité » en opposition à une vision centrée sur la biodiversité autrefois déshumanisée, la République dominicaine sa ruralité et son respect de l'environnement en opposition à un imaginaire reposant sur les stéréotypes liés à la plage et aux îles Caraïbes. 


\section{BIBLIOGRAPHIE}

ACOMUITA, “Tour Tsirutami.”, http://acomuita-costarica.jimdo.com/español/tour-tsirutami/, page consultée le 14 octobre 2018.

Acuña, Marvin et Ruiz, Keynor, "Contribución del desarrollo turístico sobre el empleo rural o agrícola en Costa Rica.” Economía y Sociedad, vol. 5, n 14, 2000, p. 85-107.

Alvarado, Jennifer et Oddone, Nahuel, “Fortalecimiento de la cadena de valor de turismo en Pedernales (República Dominicana)”, in Nahuel Oddone et Ramón Padilla Pérez (dir.), Fortalecimiento de cadenas de valor rurales, Santiago, Nations Unies, 2017, p. 243-293.

Andreu Nel-lo, Marta, “Organización y Características Del Turismo Rural Comunitario En Costa Rica.", Anales de Geografía de La Universidad Complutense, vol. 28, nº 2, 2008, p. 167-188.

Área de Planificación Regional, Ministerio de Planificación Nacional y Política Económica, Región Huerta Caribe, Plan de Desarrollo, San José, Costa Rica, MIDEPLAN, 2014, http://sia.eurosocial-ii.eu/ files/docs/1400674973-Region\%20Huetar\%20Caribe.pdf.

Auzias, Dominique et Labourdette, Jean-Paul, République Dominicaine 2012, Petit Futé, 2011, p. 9. Barel, Michel, Qualité du cacao : L'impact du traitement post-récolte, Editions Quae, 2013 p. 1-10. Barrera, Ernesto et Olivia Bringas Alvarado, « Rutas Alimentarias : una estrategia de negocios inclusivos que vincula las políticas agrarias y turísticas », Études caribéennes, n 13-14, 15 décembre 2009, http://journals.openedition.org.ezproxy.univ-paris3.fr/etudescaribeennes/3828 page consultée le 14 juin 2018.

Barrientos, Stephanie, Gender Production Networks Sustaining Cocoa-Chocolate Sourcing in Ghana and India, rapport de recherche, $\mathrm{n}^{\circ}$ 186, Manchester, Université de Manchester, Brooks World Poverty Institute, 2013.

BCRD. “Estadísticas Económicas.” 2016. http://www.bancentral.gov.do/estadisticas_economicas/ real/, page consultée le 19 septembre 2016.

Blanco, Marvin, “El agroturismo En Costa Rica. Situación y perspectivas, ECAG Informa, n 40, 2007, p. 27.

-_-.. "El Agroturismo Como Diversificación de La Actividad Agropecuaria y Agroindustrial", Desarrollo de Los Agronegocios y La Agroindustria Rural En América Latina y El Caribe. Conceptos, Instrumentos y Casos de Cooperación Técnica, IICA, San José, Costa Rica, 2010, p. 117-25.

---. 2012. "La Activación de Los SIAL Vía El Agroturismo : Análisis Del Potencial de Articulación En Cuatro Territorios Queseros de América Latina." Agroalimentaria, vol. 18 n 34, p. 123-131.

Boukhris, Linda, Imaginaire National et Imaginaire Touristique Au Costa Rica : Le Tourisme Comme Fabrique Du Territoire et de La Nation, Thèse de Géographie, Université Paris 1 Panthéon Sorbonne, 2013, http://www.theses.fr/2013PA010702, page consultée le 12 juin 2018.

-_- "La fabrique circulatoire d'un patrimoine national ou la coproduction de la nature au Costa Rica", Autrepart, n 78-79, 2016 p. 257-75. https://doi.org/10.3917/autr.078.0257, page consultée le 12 octobre 2018.

Breton, Jean-Marie, “Aires protégées, gestion participative des ressources environnementales et développement touristique durable et viable dans les régions ultra-périphériques”, Études 
caribéennes, $\mathrm{n}^{\circ}$ 12, 2009, p. 1-12 https://doi.org/10.4000/etudescaribeennes.3560, page consultée le 1 février 2017.

Caribeans Chocolate, "Chocolate Tour Puerto Viejo", Caribeans, http://

www.caribeanschocolate.com/chocolate-tour-sustainable-farming-cacao/, page conultée le 14 octobre 2018.

Ramírez Castellanos, Edwar David, “Agroecoturismo : aportes para el desarrollo de una tipología turística en el contexto latinoamericano.” Turismo y Sociedad, vol. 15, 2014, p. 223-36, https:// doi.org/10.18601/01207555.n15.13, page consultée le 8 février 2017.

Clearco Gí, Tour de Chocolate - Hato Mayor Republica Dominicana 1/2, Crearco Art Studio, Hato Mayor, 2011a, https://www.youtube.com/watch ?v =Tt9xvlLRQj0, vidéo consultée le 8 septembre 2016.

--- Tour de Chocolate - Hato Mayor Republica Dominicana 2/2, Crearco Art Studio, Hato Mayor, 2011b, https://www.youtube.com/watch ?v =jwHHgSJrceg, vidéo consultée le 8 septembre 2016.

Cochet, Hubert, L'agriculture comparée, Quae, 2011

Couve, Caroline et Laurent Croguennec, Analyse Téchnico-Économique de La Production et de La Commercialisation Du Cacao Dans La Région de San Francisco de Marcoris, République Dominicaine, Mémoire d'Agronomie Tropicale, ENSA - CNEARC, 2000. http://agritrop.cirad.fr/476996/1/ ID476996.pdf, page consultée le 19 août 2016.

De Myttenaere, Bernard, “Tourisme Rural et Valorisation Des Ressources Alimentaires Locales : Le Cas de l'AOP Fromage de Herve." Bulletin de La Société Géographique de Liège, vol. 57, 2011, p. 37 51.

Debos, Franck, "Le partenariat « viticulteurs-institutionnels du tourisme " : clé de voûte d'un œnotourisme performant", Market Management, vol. 8, n² 2, 2008, p. 62-73. https://doi.org/ 10.3917/mama.052.0062, page consultée le 21 février 2017.

Dehoorne, Olivier, "Le tourisme dans la Caraïbe. De la nécessité de sortir du tourisme de masse", Téoros. Revue de recherche en tourisme, vol. $26 \mathrm{n}^{\circ}$ 1, 2007, p. 1-5, http://journals.openedition.org/ teoros/2822, page consultée le 17 mai 2018.

-_- "Tourisme et lutte contre la pauvreté : opportunités et défis." Études caribéennes, $n^{\circ}$ 24-25, 2014, p. 1-16, https://doi.org/10.4000/etudescaribeennes.6601, page consultée le 5 février 2017.

Dehoorne, Olivier, Christelle Murat et Nathalie Petit-Charles, "International Tourism in the Caribbean Area : Current status and Future Prospects", Études Caribéennes, n 16, 2010, p. 3 , http://etudescaribeennes.revues.org/4713, page consultée le 16 juin 2018.

Dehoorne, Olivier, Pascal Saffache et Dominique Augier., "Tourisme, écotourisme et stratégies de développement dans la Caraibe", Études caribéennes, n 6, 2007 https://doi.org/10.4000/ etudescaribeennes.3383, page consultée le 14 février 2017.

Despréaux, Denis, et Agnès Leblond, "Synthèse Des Rencontres Cacao : Les Différents Aspects de La Qualité”, Plantations, Recherche, Développement, vol. 3, n², 1996, p. 113-123, https://agritropprod.cirad.fr/388351/1/document_388351.pdf., page consultée le 17 août 2016/

Dirección Regional Huerta Atlántica, Ministerio de Agricultura y Ganaderia, Desarollo cacaotero y diversificación productiva en Talamanca, MAG, 2011 http://www.mag.go.cr/acerca_del_mag/ programas/sixaola-proy02-CR-Cacao.pdf, page consultée le 18 février 2017.

Dulcire, Michel et Gille Roche, "La Co-Construction d'un Produit Territorialisé. Le Cas d'une Filière Cacao En Équateur." 3ème colloque international du réseau SYAL «Systèmes Agroalimentaires 
Localisés » Alimentation et Territoires « ALTER 2006 », Baeza, Jaen, Espagne, 18-21 octobre 2006, http://syal.agropolis.fr/ALTER06/pdf/actes/c71.pdf, page consultée le 6 juillet 2016.

Fair Labour Association, Evaluer La Situation Actuelle Des Femmes et Des Jeunes Agriculteurs et l'état Nutritionnel de Leurs Famille Dans Deux Communautés Productrices de Cacao En Côte d'Ivoire, GISCO, 2015, https://docplayer.fr/15038411-Evaluer-la-situation.html, page consultée le 16 octobre 2016.

Fairtrade International (FLO), Our Standards, https://www.fairtrade.net/standards/ourstandards.html, page consultée le 15 octobre 2018.

FAO, "Evolution de Production de Cacao de 1961 à 2014", FAOSTAT, 2014, http://faostat3.fao.org/ compare/F, page consultée le 15 mai 2016.

"Evolution de La Production de Cacao et Café En RD et Au CR", FAOSTAT, 2017. http:// www.fao.org/faostat/fr/\#compare, page consultée le 15 mai 2016.

FLO, “Cocoa." Fairtrade, 2011, http://www.fairtrade.net/products/cocoa.html, page consultée le 31 août 2016

Gasco, Janine, "The social and economic history of cacao cultivation in colonial Soconuscos, New Spain", in Alex Szogyi (dir.) Chocolate: Food of the Gods, Hofstra University, Greenwood Publishing Group, 1997.

Gobierno de Costa Rica, decreto N ${ }^{\circ}$ 39230-C-MAG , "La Gaceta.", journal officiel, n² 204, 2015, p. 5. Griotte, "Sur La Route Du Cacao." Griottes, Palette Culinaire, 2013. http://www.griottes.fr/sur-laroute-du-cacao.

Guehi, Tagro S., Adjéhi T. Dadie, Kouadio P. B. Koffi, et al., "Performance of Different Fermentation Methods and the Effect of Their Duration on the Quality of Raw Cocoa Beans: Fermentation's Effect on the Raw Cocoa Quality", International Journal of Food Science \& Technology, vol. 45, 2010, p. 2 508-2 514.

Hébert, Anne et Pascale Ammae-Khodja, Les mondes du Cacao, Cirad, 1999.

Herrera, Rémy, “Tourisme et développement dans les Caraïbes. Le cas de Cuba”, Mondes en développement, no. 157, 2012, p. 47-66, http://www.cairn.info/resume.php?

ID_ARTICLE=MED_157_0047, page consultée le 7 février 2017.

ICCO, “Fine or Flavour Cocoa.", ICCO, 2015, http://www.icco.org/about-cocoa/fine-or-flavourcocoa.html, page consultée le 13 juin 2018.

Idir, Mohamed Sofiane, Valorisation du patrimoine, tourisme et développement territorial en Algérie : cas des régions de Béjaïa en Kabylie et Djanet dans le Tassili n'Ajjer, Thèse de Sciences Economiques, Université Grenoble Alpes, 2013, https://tel.archives-ouvertes.fr/tel-00967954/document, page consultée le 4 février 2017.

Jacquet, Olivier et Gilles Laferté, “La Route des Vins et l'émergence d'un tourisme viticole en Bourgogne dans l'entre-deux-guerres", Cahiers de géographie du Québec, vol. 57 n 162, 2013, p. 425-444, https://doi.org/10.7202/1026527ar, page consultée le 12 février 2017.

Kanmogne, A., Y. Jannot, et J. Nganhou, "Description Concise et Analyse Des Systèmes Utilisés Dans La Région Sud Du Cameroun Pour Le Séchage Du Cacao.” Tropicultura, vol. 30, n² 2, 2012, p. 94-102, http://www.tropicultura.org/text/v30n2/94.pdf, page consultée le 15 juillet 2017.

Kessari Dea Edgaar, Myriam-Emilie, Quels modes de gouvernance pour un système de financement efficace pour une OP et ses producteurs, Mémoire d'ingénieur en agronomie tropicale, IRC, 2008. 
Krauss, Ulrike, Claudio Arroyo, Johnny García et al., "Manejo integrado de la moniliasis (Moniliophthora roreri) del cacao (Theobroma cacao) en Talamanca, Costa Rica", Agroforesteria en las Américas, vol. 10, n 37-38, 2003, p. 52-58.

La Nación. 2016. “Cacao de Costa Rica se aferra a la calidad para mantenerse en el mercado", La Nación, San José, 6 août 2016, https://www.nacion.com/economia/negocios/cacao-de-costa-ricase-aferra-a-la-calidad-para-mantenerse-en-el-mercado/JS2PQYSCSNE5VE56OI3IQUBTSY/story/, page consultée le 12 février 2017.

Lazzarotti, Olivier, Patrimoine et Tourisme, Belin, Belin Sup Tourisme, 2011,

Loor Solórzano, Rey Gastón, Contribution à l'étude de La Domestication de La Variété de Cacaoyer Nacional d'Équateur : Recherche de La Variété Native et de Ses Ancêtres Sauvages, Thèse de Sciences Agronomiques, Université d'Evry. 2007, http://www.supagro.fr/theses/extranet/07-0029_LOORSOLORZANO..pdf, page consultée le 17 août 2016.

Llach Cordero, Leonte, Caracterización de La Agrocadena Cacao, MAG, 2007 http://www.mag.go.cr/ bibliotecavirtual/biblioteca_vvirtual/a00083.pdf, page consultée le 15 mars 2018.

Lotero Contreras, Jorge, Mónica Liliana Arcila, et Alix Bibiana Gómez Vargas,. "Políticas públicas y turismo en las regiones rurales de Antioquia, Colombia : aproximaciones desde el enfoque de sistemas productivos territoriales y redes institucionales", Cuadernos de Desarrollo Rural, vol. 5, $\mathrm{n}^{\circ}$ 61, 2008. http://bibliotecadigital.udea.edu.co/dspace/handle/10495/3162, page consultée le 29 mai 2018

Moity-Maïzi, Pascale et Rémi Bouche, "Ancrage Territorial et Hybridation Des Savoir-Faire Au Sein d'un Système Agroalimentaire Localisé. Le Cas Des Fromages Corses”, Économie Rurale, no. 2, 2011, p. 24-38, http://www.cairn.info/resume.php?ID_ARTICLE=ECRU_322_0024, page consultée le 14 août 2016 .

Ministerio de Agricultura y Ganaderia, Mapa de Las Áreas de Producción, 2007, http:// www.mag.go.cr/acerca_del_mag/estructura/oficinas/prog-nac-cacao.html\#HERMES_TABS_1_2, page consultée le 8 juin 2018.

Ministero de Turismo “Misiones." Page officielle, Ministerio de Turismo dominicano, 2018, http:// mitur.gob.do/quienes-somos/, page consultée le 6 juin 2018.

MINTUR-DDP, Mapa de la planificación turística, 2011, http://dpp-sectur.gov.do/uploads/images/ Mapas\%20DPP/CARTOGRAFIA_DPP-MITUR-01.jpg, page consultée le 2 juin 2018.

Morera, Carlos “Concepto y Realidad Del Turismo Rural En Costa Rica”, Ambientico, n 150, 2006, p. 4-9, http://www.ambientico.una.ac.cr/pdfs/ambientico/150.pdf\#page=4, page consultée le 8 février 2018 .

Oxfam, Les Femmes et Le Cacao : Histoires d'inégalités, Oxfam France, 2013. https://

www.oxfamfrance.org/actualites/face-cachee-des-marques/femmes-et-cacao-histoiresdinegalites.

Ramirez, Pilar Emilio, Cadena de Valor Del Cacao Orgánico En La República Dominicana, Saint Domingue, 2013.

Requier-Desjardins, Denis, “Tourisme rural et développement local durable”, in Geraldine Froger (dir.), Tourisme durable dans les Suds ?, Ecopolis n 11, P.I.E. Peter Lang, 2011.

"Systèmes Agroalimentaires Localisés et Qualification : Une Relation Complexe", in VI Congreso Internacional Sistemas Agroalimentares, Florianopolis, 2013, http://nmdsc.paginas.ufsc.br/ files/2011/05/Requiers_Desjardins_Systemes_agroalimentaires_localises_et_qualification.pdf, page consultée le 19 juillet 2017. 
Rizek S.A., “Cacao Tour”, El Sendero Del Cacao, 2016, http://www.cacaotour.com/index.php/fr/ cacaotours-fr, consultée le 8 juin 2018.

Roche, Gilles, "Impact du commerce équitable sur les revenus des agriculteurs : le cas de CONACADO en République Dominicaine”, in 2ème Colloque International sur le Commerce Equitable, CIRAD-TERA, Montréal, 2006, http://www.crsdd.uqam.ca/Pages/docs/pdfColloques/ colloque_international/Seance_13/ROCHES\%202006.pdf, page consultée le 12 juin 2016.

Sarrasin, Bruno, Olivier Dehoorne et Dominique Augier, "Tourisme et ressources naturelles, prendre la mesure du défi”, Études caribéennes, n 33-34, 2016, http://

etudescaribeennes.revues.org.fr/9489, page consultée le 6 février 2017.

Sarrasin, Bruno, Élyse Lacoste-Bédard et Dominique Augier, "Le parc amazonien de Guyane française et la mise en tourisme de la nature. L'intégration de la cogestion adaptative", Études caribéennes, $\mathrm{n}^{\circ}$ 33-34, 2016, https://doi.org/10.4000/etudescaribeennes.8833, page consultée le 2 février 2017.

Tribunal Supremo de Elecciones, Aprobación Del Convenio Internacional Del Cacao, 2010. Décret $\mathrm{n}^{\circ}$ 9359, La Gaceta, Journal officiel, vol. 64, 2016, https://dialnet.unirioja.es/servlet/articulo? codigo $=3214138$, page consultée le 2 février 2017 .

UTZ, The Role of Certification and Producer Support in Promoting Gender Equality in Cocoa Production, Oxfam, 2009, https://www.utz.org/wp-content/uploads/2015/12/Gender-Position-PaperUTZ.pdf, page consultée le 22 septembre 2016.

World Cocoa Foundation, Qualité Du Cacao : Reconnaitre et Renforcer Le Rôle Des Femmes, http:// genderandcocoalivelihoods.org/fact/cocoa-quality-2/?lang=fr, page consultée le 15 septembre 2016

\section{NOTES}

1. Pour caractériser un système agraire, quatre étapes méthodologiques sont privilégiées en agriculture comparée: l'analyse du paysage, l'approche historique, l'étude des systèmes de culture puis celle des systèmes de production (Cochet H., 2011). Le tourisme est l'un des composants des systèmes de production, au même titre que le système de culture, c'est grâce au diagnostic agraire et l'agriculture comparée que l'importance du tourisme a été mise en évidence lors du premier terrain en 2011.

2. S'en sont suivis alors une quarantaine d'entretiens approfondis par pays avec les différents acteurs concernés (producteurs et productrices, femmes de producteurs, intermédiaires, transformateurs, organismes d'exportations, revendeurs, chercheurs académiques, membres des ministères concernés, agents de tourisme, touristes) mais également la participation à plus d'une dizaine de tours du cacao ainsi qu'une expérience professionnelle sur l'une des offres proposées.

3. Les cacaos reconnus spéciaux ou fins reposent sur plusieurs attributs de qualités : les attributs géographiques et organoleptiques pour les appellations d'origine; les attributs environnementaux et sanitaires pour les cacaos biologiques; les attributs sociaux pour le commerce équitable ; les attributs techniques pour les normes ISO.

4. Entretien Mariela Leandro, chercheuse du programme d'amélioration génétique du cacao et de PennState University et Wilbert Phillips, ancien directeur du programme d'amélioration génétique du cacao 2018.

5. Dirección regional Huerta Atlantica (2011: 16) ; Área de Planificación Regional (2014); Lloch Cordero, L. ; (2007).

6. Notamment Journal of Sustainable Studies ou encore Annals of Tourism Research. 
7. Cet accord démontre de la volonté des Etats à investir et développer le secteur afin de s'insérer dans le commerce international.

8. $9 \%$ du PIB résulte du tourisme selon la Banque centrale de République dominicaine (BCRD, 2016).

9. Par qualité sociale, j'entends les attributs sociaux définis par (Barel 2013:10) qui rendent compte d'un produit éthique, respectant les droits humains, valorisant les petits producteurs pour le commerce équitable (Fairtrade International [FLO], etc.

10. Il est compliqué de donner les chiffres exacts car ils dépendent de la fréquentation annuelle et du montant attribué aux différents acteurs. Néanmoins, dans le cas d'un producteur de la Conacado en 2011, j'ai pu estimer qu'il a reçu environ 500 dollars dans l'année pour faire visiter sa plantation lors du Tour del Chocolate.

11. Entretien avec Georges Ebanks, 2018.

12. Elles sont peu accessibles pour une excursion hors d'un hôtel tout inclus car trop éloignées géographiquement.

\section{RÉSUMÉS}

Le tourisme est un outil d'affichage des sociétés dominicaine et costaricienne. L'imaginaire touristique lié à la République dominicaine repose sur un tourisme de plage, tandis que celui du Costa Rica mise sur un tourisme de nature. Néanmoins, il existe aussi à présent des initiatives de tourisme rural dans les deux pays. Cet article propose d'analyser les mutations engendrées par ce type de tourisme à travers la mise en tourisme du cacao. Le tourisme du cacao est utilisé dans les deux cas comme une vitrine de la qualité du cacao. La comparaison proposée appréhende les stratégies de développement de la filière cacao et dans quelle mesure elles contribuent à des mises en tourisme du cacao différenciées. Au Costa Rica, c'est sous l'impulsion des politiques publiques que celles-ci se développent, associées à la reconnaissance du cacao comme patrimoine national. En République dominicaine, c'est le secteur privé et plus particulièrement les grands organismes exportateurs qui en sont à l'origine. Cela reflète des stratégies différentes de valorisation et de promotion, à la fois du cacao lui-même mais également d'un nouveau type de tourisme. Ce dernier permet notamment d'aller vers une plus grande visibilité de la place des femmes dans la filière. Il vient en outre bousculer les imaginaires touristiques et nationaux des deux pays et en révéler certains aspects méconnus.

El turismo es una herramienta de promoción de las sociedades dominicana y costarricense, la percepción turística vinculada con la República Dominicana se basa en un turismo de playa mientras que la de Costa Rica se basa en un turismo de naturaleza. Sin embargo, hoy en día, existe la iniciativa de turismo rural en ambos países. Este artículo ofrece analizar las mutaciones engendradas por este tipo de turismo a través de la puesta en turismo del cacao. Por ende, la comparación ofrecida captura las estrategias de desarrollo del sector cacao y en qué medida contribuyen a puestas en turismo del cacao diferenciadas. El turismo del cacao es entonces utilizado como una vitrina de la calidad del cacao. En Costa Rica, es desarrollado mediante el impulso de las políticas públicas, y el cacao es por ende reconocido como patrimonio nacional. En República Dominicana, es incentivado por el sector privado y especialmente los grandes organismos exportadores. Esto permite reflejar diferentes estrategias de valorización y de promoción, del cacao en sí, pero también de otro tipo de turismo. Este último permite 
especialmente ir hacia una mayor visibilidad de la posición de las mujeres en el sector. Viene entre otras cosas a revolucionar las percepciones turísticas y nacionales de ambos países al revelar algunos aspectos desconocidos.

Tourism is a tool used to display the cultures of both Dominican and Costa Rican societies. When tourists picture the Dominican Republic, they imagine fancy resorts and white sand beaches, and lush rainforests and nature-based activities in Costa Rica. Nevertheless, there are now rural tourism initiatives in both countries. This article proposes to analyze the mutations engendered by this type of tourism through cocoa tourism. Thus, the proposed comparison displays the development strategies of the cocoa sector and to what extent they contribute to differentiate cocoa tourism. Cocoa tourism is then used to showcase the quality of cocoa produced in these countries. In Costa Rica, it is under the impetus of public policies that cocoa tourism is encouraged, allowing cocoa to be recognized as national heritage. In the Dominican Republic, it is the private sector and especially the major exporting organizations that implement it. This reflects different strategies of valorization and promotion, not just cocoa but also of another type of tourism. Cocoa tourism has allowed women to be recognized for their accomplishments, as they not only have the opportunity to run their own cocoa business, but also showcase their endeavors to the public. It also changes the tourist and national imaginary of the two countries by revealing some unknown aspects.

\section{INDEX}

Mots-clés : Tourisme, Cacao, République dominicaine, Costa Rica, Tourisme rural

Palabras claves : Turismo, Cacao, Republica Dominicana, Costa Rica, Turismo rural

Keywords : Tourism, Cacao, Dominican Republic, Costa Rica, Rural tourism

\section{AUTEUR}

\section{LAURA HENRY}

Doctorante contractuelle en Géographie, IHEAL, Université Sorbonne Nouvelle - Paris 3Ingénieure en agro-développement international, ISTOM. Coordinatrice du pôle Amérique centrale de l'Institut des Amériques. Centre de Recherche et de Documentation sur les Amériques (CREDA-UMR 7227), Université Sorbonne Nouvelle - Paris 3. laura.henry.istom@gmail.com // pole.ameriquecentrale@institutdesameriques.fr 